\title{
Profili costituzionali della tutela dei diritti sui beni immateriali
}

\section{(Das Immaterialguiterrecht und die italienische Verfassung)}

Carola Pagliarin

\section{La tutela dei diritti sui beni immateriali e principi costituzionali}

Come è noto, nella nostra Costituzione non si rinvengono norme dedicate in modo espresso alla tutela dei diritti sui beni immateriali o della proprietà intellettuale. Non si sono, dunque, seguiti precedenti illustri, come la Costituzione americana del $1787 .^{1}$

Ciò non di meno sussiste certamente, come si vedrà, un fondamento costituzionale di una tale tutela e su di esso risulta utile riflettere per una molteplicità di ragioni.

In primo luogo, perché questo ci impone di riflettere sulle finalità degli istituti e, dunque, sulla loro matrice e sulla loro adeguatezza rispetto al presente.

In secondo luogo, in quanto ci porta a collocare gli stessi istituti nell' ambito del sistema dei valori costituzionali, presupposto per confrontare poi la prospettiva interna con la disciplina internazionale ed europea, di cui altri si occuperanno nel presente volume.

Ancora - e soprattutto - perché ci costringe a ragionare in una dimensione storica e dinamica, particolarmente preziosa in un'epoca di mutamenti repentini e radicali.

Le riflessioni che si svolgeranno hanno, dunque, la finalità di fornire qualche spunto di riflessione e uno sguardo di prospettiva.

\footnotetext{
${ }^{1}$ The Constitution of the United States - 1787 - „Section VIII: 1. The Congress shall have power (...) 8. To promote the progress of science and useful arts by securing for limited times to authors and inventors the exclusive right to their respective writings and discoveries".
}

\footnotetext{
C. Pagliarin $(\varangle)$

Dipartimento di Diritto Pubblico, Internazionale e Comunitario, Università degli Studi di Padova, Padua, Italien

E-Mail: carola.pagliarin@studiolegalepmb.com
} 
Ora, come si è appena ricordato, la Costituzione italiana non prevede espressamente la tutela dei diritti sulle opere dell'ingegno.

Tuttavia, diversi disposti costituzionali possono essere - e sono in effetti stati invocati a fondamento di tale tutela, non senza difficoltà, contraddizioni e ambivalenze nella loro identificazione.

La cornice costituzionale in cui inserire la tutela dei diritti sui beni immateriali si presenta tutt'altro che nitida, soprattutto se si desidera ragionare in un'ottica di insieme, senza scendere alle peculiarità delle diverse fattispecie.

Del resto, la stessa individuazione dei beni immateriali trae origine da una comunità di giuristi - la scuola della Pandettistica tedesca - fortemente incline alla concettualizzazione dell'esperienza giuridica, al punto da sbiadire le eterogeneità socioeconomiche, oltre che le stesse differenze di trattamento giuridico. ${ }^{2}$

Solo per dar ingresso alla complessità della trama dei principi e dei valori, si possono qui richiamare alcuni dei titoli legittimanti la tutela di cui si discute, che poi si andranno ad analizzare anche alla luce della giurisprudenza costituzionale.

Così viene in rilievo l'art. 2 Cost., in quanto tutela i diritti inviolabili dell'uomo, tra cui viene ricompreso il diritto dell' autore ad essere riconosciuto tale rispetto alla propria opera dell'ingegno ed il relativo diritto di esclusiva sul suo sfruttamento economico. Tuttavia, lo stesso disposto, come si cercherà di illustrare oltre, può essere evocato per porre dei limiti al medesimo diritto, in ragione dei doveri inderogabili di solidarietà economica e sociale, ivi ricordati.

Si possono invocare poi l'art. 42 Cost., che tutela la proprietà, in tutte le sue forme, o - valorizzando l'impostazione fatta propria dal legislatore ordinario italiano nel Codice civile - l'art. 4 Cost. e l'art. 35 Cost., che tutelano il lavoro,,in tutte le sue forme e applicazioni“", nella cornice del più ampio principio lavoristico proprio della Costituzione italiana, di cui è fondamento l'art. 1 Cost. Se assolutizzate queste prospettive sono state, a volte, giudicate parziali e insufficienti, altre volte, finanche contraddittorie.

Altre previsioni costituzionali, invece, presentano un'intrinseca ambivalenza rispetto al fondamento costituzionale della tutela dei diritti sui beni immateriali.

Si pensi all'art. 9 Cost., secondo cui la Repubblica promuove lo sviluppo della cultura e la ricerca scientifica e tecnica, all'art. 21 Cost., che, proclamando la libertà di espressione, consente l'esplicazione dell'attività di creazione e divulgazione dell'opera o all'art. 33 Cost., sulla libertà dell'arte e della scienza.

In queste ultime disposizioni costituzionali possiamo veder sanciti diritti e libertà che consentono il realizzarsi delle opere dell'ingegno, ma gli stessi diritti e libertà potrebbero essere limitati proprio dagli istituti di protezione degli autori e da tutti i sistemi di esclusiva.

\footnotetext{
${ }^{2}$ Come osserva P. Spada, Parte generale, in AA.VV., Diritto industriale. Proprietà intellettuale e concorrenza, Torino, 2012, 6 ss. I beni immateriali sono stati ricatalogati nei „nuovi beni“ (così S. Praduroux, Diritto di proprietà (come diritto fondamentale), in Digesto delle Discipline Privatistiche, Torino, 2012) e accostati all'espressione „nuovi modi di possedere“, riferita alla proprietà collettiva da P. Grossi, Un altro modo di possedere. L'emersione di forme alternative di proprietà alla coscienza giuridica postunitaria, Milano, 1977, riprendendo il pensiero di Carlo Cattaneo.
} 
Emerge, dunque, uno strutturale e peculiare conflitto di interessi, che tradizionalmente viene risolto in concreto dal legislatore, nel dettare la disciplina dei diritti sui beni immateriali.

Diritti che, proteggendo gli autori, incentivano il processo creativo e innovativo con i relativi investimenti economici, ma che sono stati considerati di ostacolo al progresso della ricerca e allo sviluppo o, anche, semplicemente, all'esercizio di libertà.

Ancora, il sistema delle esclusive può persino entrare in contrasto concreto con la tutela di diritti fondamentali, come il diritto alla salute - di cui all'art. 32 Cost. -, quando incidano sui costi delle prestazioni non fungibili che realizzano i diritti sociali.

Se il contrasto non si pone necessariamente in linea teorica, si presenta però in concreto, in un sistema come il nostro di scarse risorse pubbliche e con un quadro demografico particolarmente negativo, che vede un peso sempre crescente, in termini percentuali, della popolazione anziana, bisognosa di cure, su quella attiva, generatrice di reddito.

Viene, infine, in rilievo il complesso disposto dell'art. 41 Cost, sulla libertà di iniziativa economica e suoi limiti, con tutta la carica della sua intrinseca ambiguità. ${ }^{3}$

Da tempo ormai entra in gioco la discussa dialettica tra tutela della concorrenza che oggi ha un esplicito e diretto fondamento costituzionale nell'art. 117 Cost. - e la tutela della proprietà intellettuale.

Si tratta di un tema tipico del diritto sovranazionale ed europeo, che vede non di rado posizioni, anche istituzionali, che negano il contrasto tra la tutela dei diversi valori in campo, in una visione armonica, che rischia però di soffrire di un certo astrattismo.

Nonostante i riferimenti alla Carta fondamentale appaiano tutt'altro che univoci, proprio la prospettiva o, se si vuole, gli ,strumenti“ del diritto costituzionale classico possono fornire spunti per una ricostruzione sistematica delle questioni poste.

\section{Il contrasto tra diritti e interessi nel bilanciamento dei diritti}

Va ricordato che i diritti e le libertà costituzionali sono espressi come principi, dunque attraverso norme dotate di un elevato grado di genericità, e sono affermati in linea tendenziale senza gerarchie o precedenze. ${ }^{4}$

\footnotetext{
${ }^{3}$ Si tratta di una caratteristica sempre sottolineata del disposto costituzionale citato. A tale riguardo basti ricordare le lucide riflessioni già a margine del processo costituente di P. Calamandrei, Come nasce la nuova Costituzione, in Il Ponte, 1946: „Ma nel campo economico è ancora tutto affidato all'avvenire: e la Costituzione democratica italiana, invece che lo specchio fedele e la legalizzazione formale di una rivoluzione già avvenuta nel passato, deve essere necessariamente considerata come lo strumento predisposto per rendere possibile, nell'avvenire, in forme progressive e legalitarie, quella trasformazione sociale che oggi è appena agli inizi“.

${ }^{4}$ Così, in termini generali, R. Bin - G. Pitruzzella, Diritto costituzionale, Torino, 2019, 540 ss.
} 
Nella Carta costituzionale i diritti fondamentali non sono mai affermati in termini assoluti, ma fanno parte di un medesimo tessuto complesso, in cui altri diritti e altri interessi e beni costituzionalmente protetti possono legittimamente limitarne la portata.

La Costituzione italiana è strutturalmente preordinata alla logica della relazionalità degli interessi: non si limita ad elencare i diritti, ma esplica dei nessi di collegamento, di modo che affermando i diritti ne prevede spesso i limiti. In altre parole, il testo costituzionale predispone i diritti stessi ad un dialogo e non consente di considerarli indipendenti l'uno dall'altro. ${ }^{5}$

La stessa Corte costituzionale, in una significativa pronuncia, ${ }^{6}$ ha ricordato che ,tutti i diritti fondamentali tutelati dalla Costituzione si trovano in rapporto di integrazione reciproca e non è possibile pertanto individuare uno di essi che abbia la prevalenza assoluta sugli altri" ${ }^{6.7}$ La tutela deve essere, dunque, sempre , sistemica“6, secondo una tecnica interpretativa e ricostruttiva che riflette il pluralismo dei valori su cui si fonda la Costituzione italiana. ${ }^{8}$

Altrimenti ragionando - ammonisce il giudice delle leggi -, , ,si verificherebbe l'illimitata espansione di uno dei diritti, che diverrebbe 'tiranno' nei confronti delle altre situazioni giuridiche costituzionalmente riconosciute e protette, che costituiscono, nel loro insieme, espressione della dignità della persona ${ }^{66} .9$

In ipotesi significative la Costituzione con ricchezza di disposizioni finalistiche, istituisce rapporti tra $\mathrm{i}$ diritti che appaiono di gerarchia, ma che sono pur seppur sempre di natura relazionale e non rigida. E, tuttavia, anche laddove il bilanciamento non trovi risoluzione immediata nel dettato costituzionale, rinviene comunque in quest'ultima, soprattutto nei Principi fondamentali, un orientamento decisivo e vincolante per l'interprete. ${ }^{10}$

Quando la Corte costituzionale italiana è chiamata a giudicare della legittimità del compromesso tra interessi confliggenti fissato dalla legge - il bilanciamento dei diritti - procede con argomentazioni che è difficile distinguere da quelle tipiche del giudizio di ragionevolezza ${ }^{11} \mathrm{o}$ dall' applicazione del principio di proporzionalità. ${ }^{12}$

\footnotetext{
${ }^{5} \mathrm{~F}$. Pizzolato, Il principio costituzionale di fraternità. Itinerario di ricerca a partire dalla Costituzione italiana, Roma, 2012, 138; G. Berti, Manuale di interpretazione costituzionale, Padova, 1994, 419; P. Perlingeri, La personalità umana nell'ordinamento giuridico, Napoli, 1972, 147.

${ }^{6} \mathrm{Si}$ fa riferimento alla sentenza n. 85 del 2013 sul caso ILVA di Taranto.

${ }^{7}$ In altra pronuncia - nella sentenza n. 170 del 2013 - la Corte costituzionale include in questo approccio integrato ai diritti fondamentali anche i diritti protetti dalla CEDU, non già per l' ,,affermazione della primazia dell'ordinamento nazionale“, ma per l' „integrazione delle tutele“.

${ }^{8}$ In tema R. Bin, Ragionevolezza e divisione dei poteri, in M. La Torre e A. Spadaro (a cura di), La ragionevolezza nel diritto, Torino, 2002, 59 ss.

${ }^{9}$ Corte cost. sent. n. 85 del 2013, sopra citata.

${ }^{10} \mathrm{~F}$. Pizzolato, Il principio costituzionale di fraternità cit., 139.

${ }^{11}$ Per tutti si veda L. Paladin, Ragionevolezza (principio di), in Enc. Dir., Aggiornamento, I, Milano, 1997, 899 ss. Va ricordato, del resto, che, a sua volta, il principio di ragionevolezza è nato nell'ambito dei giudizi sul principio di eguaglianza, ma da questo si è progressivamente emancipato. In tema F. Sorrentino, Eguaglianza formale, in Costituzionalismo.it, n. 3/2017.

${ }^{12}$ Sottolinea questa intrinseca indeterminatezza nell'utilizzo da parte della Corte italiana di categorie concettuali in sé diverse M. Cartabia, I principi di ragionevolezza e proporzionalità nella giurisprudenza costituzionale italiana, in www.cortecostituzionale.it, 2013.
} 
Il bilanciamento a cui si fa riferimento, come è noto, è una tecnica impiegata in genere da tutte le corti costituzionali per risolvere questioni di costituzionalità in cui si registri un contrasto tra diritti e interessi diversi (balancing test, nella terminologia nord-americana, Abwägung in quella tedesca), ${ }^{13}$ tuttavia, nei giudizi svolti dal nostro giudice delle leggi presenta tratti caratteristici peculiari non solo in ragione dell'incertezza nella terminologica utilizzata, ${ }^{14}$ ma anche per la struttura dell'argomentazione seguita.

Così la Corte costituzionale italiana non opera - in ultima analisi - alcuna distinzione tra principio di ragionevolezza e principio di proporzionalità, ${ }^{15}$ i quali sono spesso usati in modo del tutto fungibile l'uno rispetto all'altro. ${ }^{16}$

Nelle pronunce costituzionali si pone mente alla correttezza del bilanciamento legislativo alla luce del parametro costituzionale. L'indagine circa il livello di protezione di determinati interessi e la loro ragionevole ponderazione si presta ad una lettura in chiave deontologica: si risolve, cioè, nella ricerca di una sistemazione degli interessi potenzialmente alternativa alla ricostruzione legale e, quindi, causa di illegittimità della legge. ${ }^{17}$

Per individuare criteri generali di ordinazione del conflitto degli interessi coinvolti è necessario preliminarmente individuare gli interessi compresenti nell' ambito tematico in oggetto e qualificare il livello di protezione formale: occorre tracciare insomma la ,topografia del conflitto““.18

Possono essere enucleate regole di bilanciamento procedimentali, formali ${ }^{19}$; regole generali come quelle della coessenzialità e proporzionalità del limite, alla stregua delle quali le posizioni giuridiche tutelate come diritti fondamentali possono legittimamente essere compresse solo se ciò risulti indispensabile e non altrimenti evitabile per realizzare un altro interesse costituzionalmente rilevante e comunque con i mezzi strettamente necessari per il conseguimento del fine. Tuttavia, come si

\footnotetext{
${ }^{13}$ R. Bin - G. Pitruzzella, Diritto costituzionale cit.

${ }^{14}$ L. Paladin, Ragionevolezza (principio di) cit.; Id., Esiste un ,principio di ragionevolezza“ nella giurisprudenza costituzionale?, in Il principio di ragionevolezza nella giurisprudenza della Corte costituzionale, Milano, 1994 in cui si afferma - nettamente - che un principio di ragionevolezza non esiste, dato che si tratta di una formula verbale alla quale non corrisponde un concetto o una nozione ben determinata.

${ }^{15}$ Sulla struttura del giudizio di proporzionalità, per tutti, A. Barak, Proportionality, Cambridge, 2012.

${ }^{16} \mathrm{M}$. Cartabia, I principi di ragionevolezza e proporzionalità cit.

${ }^{17} \mathrm{G}$. Scaccia, Controllo di ragionevolezza delle leggi e applicazione della Costituzione, in Nova juris interpretatio, Roma, 2007, 286 ss; Id., Gli strumenti della ragionevolezza nel giudizio, Torino, 2000. Che sottolinea l'effetto di indebolimento del testo costituzionale che il ricorso al principio di ragionevolezza determina, ma, al contempo, il potenziamento della normatività della Costituzione che lo stesso ad un tempo produce.

${ }^{18} \mathrm{R}$. Bin, Diritti e argomenti. Il bilanciamento degli interessi nella giurisprudenza costituzionale, Milano, 1992, 62 ss.

${ }^{19}$ Sul punto G. Scaccia, Gli „strumenti“ della ragionevolezza nel giudizio costituzionale, Milano, 2000, 348 ss.
} 
è già sottolineato, risulta del tutto estranea all'esperienza italiana l'elaborazione e la sistematizzazione di una sequenza di standard di giudizio disposti in progressione, diffusi, invece, in altre esperienze, compresa quella europea. ${ }^{20}$

Quello che, dunque, insegna il diritto costituzionale è che ricercare l'astratta armonia tra principi e valori a poco serve perché nel terreno concreto della vita sociale ed economica il conflitto tra diritti e interessi di rilievo costituzionale è fisiologico. Negare il conflitto significa lasciare che la decisione sulla prevalenza o sul punto di contemperamento avvenga sotto traccia, in modo non dichiarato.

L'ordinazione degli interessi che qualsiasi legislatore compie - e che qualsiasi corte costituzionale poi rinviene e verifica - è sempre insidiosa, proprio perché in essa vi è un sacrificio, sia pure parziale, di diritti e libertà. La scelta a fondamento della disposizione, dunque, deve essere trasparente, ricostruibile e sindacabile.

Ugualmente, è essenziale che il giudizio di bilanciamento del giudice delle leggi sia sorretto da una motivazione adeguata, che ne consenta lo scrutinio logico e giuridico, non potendosi di certo trasformare in arbitrio. ${ }^{21}$

\section{Il bilanciamento in tema di diritti sui beni immateriali. Un'ipotesi emblematica: la brevettazione dei farmaci}

Questa tecnica di bilanciamento è stata, ad esempio, utilizzata dalla Corte costituzionale già nella sentenza n. 20 del 1978 per giudicare della legittimità costituzionale del divieto di brevettazione dei farmaci, di cui al Regio decreto n. 1127 del 1939 (recante il ,Testo delle disposizioni legislative in materia di brevetti per invenzioni industriali“") e a lungo vigente nell'ordinamento italiano.

La pronuncia originava, in realtà, da un'inerzia legislativa prolungata. Infatti, nonostante diversi tentativi di modifica della disciplina del brevetto, effettuati fin dagli inizi del Regno d'Italia e proseguiti nei primi decenni della Repubblica, ancora negli anni ' 70 del secolo scorso permaneva il divieto del brevetto per i farmaci. La concessione della privativa industriale venne bloccata dalla mancata emanazione dei regolamenti pur previsti dalla normativa in vigore e da disposizioni con cui si

\footnotetext{
${ }^{20} \mathrm{Si}$ rinvia ancora a M. Cartabia, I principi di ragionevolezza e proporzionalità cit.

${ }^{21} \mathrm{La}$ Corte costituzionale italiana è stata spesso sospettata - con particolare riferimento alla formulazione dei giudizi di ragionevolezza e di proporzionalità - di travalicare i confini delle scelte giurisdizionali per svolgere valutazioni di merito politico. Tanto che il giudice delle leggi in più di un'occasione (cfr., ad esempio, Corte cost., sent. n. 1130 del 1988 e sent. n. 89 del 1996) ha sottolineato in modo esplicito la differenza tra giudizio di ragionevolezza e scelte di opportunità politica, proprie del legislatore. Sul punto si veda A. Anzon, Modi e tecniche del controllo di ragionevolezza, in R. Romboli (a cura di), La giustizia costituzionale a una svolta, Torino, 1991, 31 ss. M. Luciani, Lo spazio della ragionevolezza nel giudizio costituzionale, in Il principio di ragionevolezza cit., 246 ss., sottolinea come l'elaborazione di canoni di giudizio chiari, applicati in modo esplicito, alimenti la coerenza, la prevedibilità e la controllabilità delle decisioni, attraverso la motivazione, consolidando l'autorevolezza del giudice delle leggi e ponendolo al riparo dalla sovraesposizione politica.
} 
stabiliva il differimento della cessazione del divieto di brevetto. ${ }^{22}$ In ragione dell'inerzia, numerose imprese farmaceutiche di diversa nazionalità chiesero alla Commissione ricorsi contro i provvedimenti dell'Ufficio centrale brevetti la riforma di alcune decisioni che avevano negato il brevetto di prodotti di uso farmaceutico. A seguito di tali richieste, la Commissione aveva sollevato, con diciotto ordinanze analoghe, questione di legittimità costituzionale rispetto al divieto contenuto nell'art. 14 del r.d. n. 1127 del 1939.

La Corte costituzionale con la pronuncia citata ha affermato che il divieto di brevettare i medicamenti ed i processi diretti a produrli non solo risultava fondato su motivi specifici non corrispondenti più alla realtà, ma che neppure poteva dirsi congruo rispetto all'interesse generale alla tutela della salute, di cui all'art. 32 Cost.

Secondo la Consulta, esso costituiva, dunque, un'ingiustificata deroga alla par condicio degli inventori industriali, con riguardo sia al diritto personale al riconoscimento della paternità dell'invenzione, che alle situazioni economiche di vantaggio cui dà luogo l'esclusiva.

Per altro verso, ad avviso della Corte, il divieto di brevettazione penalizzava gli imprenditori del settore farmaceutico rispetto a quelli di altri settori, ed ostacolava, altresì, la ricerca scientifica e tecnica, la cui promozione costituisce, al contrario, dovere della Repubblica.

Pertanto, è stato dichiarato costituzionalmente illegittimo il comma primo dell'art. 14, r.d. 29 giugno 1939 n. 1127 per contrasto con gli artt. 3, 41 e 9 Cost., mentre sono state considerate assorbite le pur proposte censure riferite agli artt. 42 e 43 Cost.

Le argomentazioni del giudice delle leggi sono preziose per riflettere sul fondamento costituzionale della tutela dei diritti sui beni immateriali in una prospettiva di equilibrio rispetto agli altri valori costituzionali, segnatamente nella materia insieme peculiare e paradigmatica - della tutela della salute, di cui al primo comma dell'art. 32 Cost.

La sentenza n. 20 del 1978, per quanto risalente, risulta emblematica dell'utilizzo da parte della Corte costituzionale italiana di quelle tecniche di decisione, che attraverso lo scrutinio del rispetto del principio di eguaglianza, finiscono per operare il bilanciamento dei diritti e fare applicazione dei canoni di ragionevolezza, di cui si è tentato di tracciare sinteticamente l'evoluzione nelle pagine precedenti.

In questa prospettiva la Consulta ricostruisce la disciplina normativa della materia nel suo divenire storico e nelle ragioni giustificatrici non certo per amor di erudizione, ma per sottoporla allo scrutinio costituzionale.

Facendo applicazione del principio di eguaglianza, e ammessa come pacifica l'esistenza del tertium comparationis riguardo alle invenzioni nel settore farmaceutico, consistente nel più vasto genere delle invenzioni industriali, la Corte ha ritenuto che il motivo di deroga non presenti ,la nota necessaria della esclusività““.

L'irragionevolezza della scelta legislativa viene colta sia rispetto alle discipline ugualmente finalizzate alla tutela della salute, quali quelle in materia alimentare o

\footnotetext{
${ }^{22}$ Una attenta ricostruzione delle vicende normative e della dottrina in tema si trova in R. Pardolesi, Sul divieto di brevettazione di farmaci, in Foro it., 1978, I, 809.
} 
relative ai presidi medico-chirurgici, che pure prevedevano la brevettabilità, sia in relazione al venir meno delle ragioni storiche che avevano originato la deroga.

Soprattutto, la Corte si domanda ,se la disciplina derogativa sia congrua rispetto all'interesse generale quale emerge a questo proposito dalla normativa della Costituzione“". Interesse che viene individuato nella tutela della salute, a cui devono coordinarsi, ,quali mezzi al fine ${ }^{66}$, la disciplina del prezzo e della adeguata presenza quantitativa sul mercato dei farmaci oltre che ,la ricerca scientifica e tecnica organizzata nell' ambito dell'industria farmaceutica“".

Il coordinamento di questi fattori deve avvenire - osserva il giudice delle leggi in modo ,equilibrato“, non sacrificandosi ,,in misura grave nessuno di essi““.

La disciplina sottoposta a scrutinio risulta, pertanto, incostituzionale poiché finisce per sacrificare proprio ,la componente della ricerca scientifica e tecnica, essenziale ormai per assicurare l'ulteriore progresso nel settore della produzione farmaceutica“6.

La Consulta ricorda che ,una delle finalità del conferimento dei diritti patrimoniali derivanti dalla brevettazione è quella di incentivare la ricerca, coprendo innanzitutto le ingenti spese che comporta la sua organizzazione ed il suo svolgimento".

Viene qui, dunque, in considerazione, accanto alla tutela di cui all'art. 9 Cost., con la valutazione dell'investimento economico per la creazione del nuovo prodotto, la norma di cui all'art. 41 Cost. che garantisce la libertà di iniziativa economica privata, invocato da parte della dottrina come fondamento della tutela costituzionale dei diritti di cui si discute. ${ }^{23}$

La Corte svolge una valutazione della ragionevolezza dell'assetto degli interessi coinvolti sulla considerazione esplicita dell'evoluzione concreta della realtà sottostante nel suo divenire, considerando la regolazione dei prezzi, l'intervento dello Stato, il ruolo della ricerca privata e delle dinamiche di relazione con i mercati esteri.

Alla luce di tutto questo ne deduce, ,la non congruità con l'interesse generale alla tutela della salute“ della disciplina sottoposta al suo scrutinio ,ove si consideri la sproporzione tra il mezzo prescelto (divieto di brevettazione) e la possibilità di soddisfare quell'interesse con mezzi più rispettosi del principio di eguaglianza“".

La Corte, quindi, fa applicazione di quelle tecniche di decisione, non ben distinguibili nell'applicazione dei diversi criteri (bilanciamento tra diritti, proporzionalità, eguaglianza e via discorrendo), come si è sopra evidenziato, ma comunque ricostruibili all'interno del canone complessivo della ragionevolezza e al criterio della ,corrispondenza alla realtà‘.

La Consulta - secondo i suoi consueti canoni di ragionamento - considera l'esperienza, il dato di fatto, evidenziandosi un elemento concreto nella valutazione di ragionevolezza. Il giudizio sulle leggi non può risolversi in un confronto meccanico tra due regole, ma richiede di valutare la rispondenza di una legge ad un principio o a un valore. ${ }^{24}$

\footnotetext{
${ }^{23}$ P. Spada, „Creazione ed esclusiva“, trent'anni dopo, in Riv. Dir. civ., 1997, I, 215 ss.; Id., Parte generale cit., 20.

${ }^{24} \mathrm{~L}$. Mengoni, il diritto costituzionale come diritto per principi, in Studi in onore di Feliciano Benvenuti, II, Modena, 1996, 1141 ss.; G. Pino, Diritto e interpretazione. Il ragionamento giuridico nello Stato costituzionale, Bologna, 2010, 51 ss e 201 ss.; M. Cartabia, I principi cit., 17.
} 
Nell'impossibilità di fissare una volta per tutte un rapporto gerarchicamente predeterminato tra gli elementi in gioco, il giudice delle leggi opera un bilanciamento dinamico, in concreto, non astrattamente prestabilito.

In particolare, nel decidere sulla conformità a Costituzione del divieto di brevettabilità dei farmaci, basandosi implicitamente sul principio personalista, la Consulta ha riconosciuto una priorità al diritto alla salute (art. 32 Cost.), da ottenersi, quale mezzo al fine, grazie allo sviluppo di una ricerca scientifica (art. 9 Cost.), ma senza consentire gerarchie assolute o visioni monistiche.

Se la tutela della salute deve costituire l'obiettivo del legislatore, lo sviluppo della ricerca, in sé compito e valore della Repubblica, si armonizza quale veicolo di realizzazione della stessa e vede nel riconoscimento del brevetto un utile elemento strumentale, nella considerazione delle dinamiche economiche e sociali che si ponevano innanzi alla Corte al momento della decisione.

In questa prospettiva, si giustificavano una serie di possibili contemperamenti della privativa brevettuale, fra cui una durata ridotta, un regime derogatorio in forma di licenza obbligatoria, un contemperamento autoritativo in termini di congruità del prezzo, fino ad un possibile intervento di segno espropriativo.

Il giudice delle leggi, dunque, ha tracciato una via che, utilizzando gli strumenti del bilanciamento e del principio di ragionevolezza, consente al legislatore ordinario, attraverso meccanismi correttivi e di intervento, di regolare la materia della brevettazione dei farmaci nel rispetto del dettato costituzionale.

In dottrina ${ }^{25}$ si è criticamente osservato però che il brevetto del farmaco, che la Corte aveva ritenuto costituzionalmente conforme all'interno di una precisa logica che lo vedeva come strumento per raggiungere obiettivi legati al progresso della ricerca ed alla salute individuale e collettiva, possa condurre oggi a risultati distorti alla luce di casi non isolati, anche se non generalizzabili.

Si censurano così episodi - quale, ad esempio, quello legato alle note vicende del sofosbuvir, particolarmente efficace contro l'epatite C - in cui il brevetto ha condotto all'esclusione di intere categorie di malati dalle cure più efficaci, dimostrando come il mercato del farmaco rischi di essere territorio in cui dominano poteri incontrollati il cui esercizio va a concreto svantaggio dei diritti individuali.

Stante il quadro così delineato, si invoca la necessità di recuperare il significato originale e più autentico dei principi indicati dalla giurisprudenza costituzionale attraverso strumenti che, agendo nel solco della vocazione del costituzionalismo, possano permettere un controllo efficace ed una effettiva limitazione del potere (anche) economico allo scopo di tutelare e promuovere il fondamentale diritto alla salute. ${ }^{26}$

\footnotetext{
${ }^{25}$ C. Casonato, I farmaci, fra speculazione e logiche costituzionali, in Rivista AIC, 2017, n. 4, 30.12.2017.

${ }^{26} \mathrm{C}$. Casonato, I farmaci cit. Si veda al riguardo anche la mozione adottata dal Comitato Nazionale per la Bioetica italiano, in data 23 febbraio 2017, Per una politica di accesso equo a farmaci innovativi ad alta efficacia per patologie gravi: riduzione dei prezzi e contenimento dei costi a carico del SSN e dei cittadini: http://bioetica.governo.it/media/172131/m18_2017_farmaci-innovativi_it.pdf.
} 
Del resto, la necessità di un bilanciamento concreto tra interessi e valori potenzialmente confliggenti, è presente in un'altra più recente pronuncia del giudice delle leggi in tema di spesa farmaceutica.

La Corte costituzionale con la sentenza n. 70 del 2017 ha dichiarato non fondata la questione di legittimità costituzionale dell' art. 5, comma 3, lett. a), del d.1. n. 159 del 2007, conv., con modif., nella legge n. 222 del 2007, censurato dal TAR Lazio in riferimento all'art. 3 Cost. - nella parte in cui prevede che l'obbligo di ripianare il superamento del tetto della spesa farmaceutica imputabile al fondo aggiuntivo per l'acquisto di farmaci innovativi sia a carico di tutte le aziende titolari di autorizzazione all'immissione in commercio (AIC), in proporzione ai rispettivi fatturati relativi ai medicinali non innovativi coperti da brevetto.

Secondo la Consulta la disposizione censurata realizza un bilanciamento non irragionevole tra le esigenze di diffusione e promozione dell'innovazione farmaceutica - e quindi di tutela della salute pubblica - e quelle di razionalizzazione e contenimento della spesa sanitaria, in quanto obbliga le aziende produttrici di farmaci non innovativi coperti da brevetto a contribuire ad un sistema - quello della rimborsabilità dei farmaci erogati dal SSN - dal quale esse ricavano indubbi benefici. La norma, inoltre, commisurando il riparto ai rispettivi fatturati, viene ad esprimere un'esigenza di proporzionalità dell'onere sia rispetto alla solidità finanziaria dei destinatari, sia rispetto alla misura della loro partecipazione al sistema dell'assistenza farmaceutica e, in particolare, al regime di rimborsabilità dei farmaci.

Né l'incidenza dell'onere su aziende che non hanno superato il proprio budget annuale può ritenersi manifestamente irragionevole, tenuto conto dell'inidoneità strutturale del criterio dell'imputabilità dello sforamento in riferimento all' acquisto di farmaci innovativi (non rapportabile a consumi registrati nei precedenti anni di spesa), della sussistenza di un interesse pubblico a favorire lo sviluppo e la disponibilità di terapie farmacologiche innovative, della possibilità che la compartecipazione all'onere si traduca in un incentivo ad investire nell'innovazione farmaceutica e della temporaneità del meccanismo distributivo scelto. La maggiore gravosità di tale determinazione legislativa rispetto alla pluralità delle opzioni a disposizione del legislatore al fine di conseguire una ragionevole graduazione degli interessi costituzionali coinvolti, del resto, è stata rimodulata e alleggerita dall'evoluzione legislativa, attraverso un progressivo trasferimento dell' onere a carico delle stesse imprese titolari di AIC per farmaci innovativi e un rilevante incremento delle risorse destinate alla spesa per la stessa tipologia di farmaci.

In questo ampio quadro di composizione di interessi tracciato dal legislatore e non censurato dalla Consulta emerge la considerazione di una ulteriore prospettiva verso cui opera il bilanciamento.

Sempre individuando la priorità della tutela della salute su valori costituzionali che rispetto ad essa possano dirsi serventi - come la promozione della ricerca e del progresso tecnologico a cui fa riferimento il regime di brevettabilità - e, dunque, dando ancora una volta applicazione al principio personalista di cui all'art. 2 Cost., il giudice delle leggi rinviene nella trama legislativa la considerazione di interessi ulteriori a loro volta intrinsecamente connessi con i precedenti. 
La Corte costituzionale, infatti, mette implicitamente in relazione la previsione di oneri discendenti dalla titolarità del brevetto alle esigenze di equilibrio della finanza pubblica, di cui agli artt. 81, 97 e 119 Cost., che finiscono per potersi riflettere sull'effettività del diritto alla salute.

Emerge in tal modo nella disciplina di aspetti che attengono ai diritti sui beni immateriali anche la logica del bilancio pubblico. Logica che, del resto, ha trovato esplicito riconoscimento nella Carta a seguito delle modifiche dettate dalla legge costituzionale n. 1 del 2012 sul cosiddetto ,pareggio di bilancio“6. ${ }^{27}$

\section{Il fondamento costituzionale del diritto d'autore}

I criteri propri del bilanciamento dei diritti sono stati applicati anche in ordine alle numerose questioni sorte intorno al riconoscimento dei diritti dell'autore delle opere creative.

Invero, la ricerca di un fondamento costituzionale del diritto di autore, che renda costituzionalmente necessari gli elementi essenziali di quel diritto, non è stata oggetto di ampie riflessioni tra i giuristi italiani, e la stessa Corte costituzionale ha dato l'impressione di non voler affrontare la questione in modo esplicito. ${ }^{28}$

La dottrina, sviluppatasi maggiormente tra gli studiosi del diritto privato - e, segnatamente, industriale - che non tra i cultori delle discipline pubblicistiche, ha spesso toccato il tema a margine di analisi riguardanti questioni più ampie o generali.

Del resto, gli stessi risultati delle riflessioni sul fondamento costituzionale del diritto d'autore risultano determinati dall'evoluzione delle modalità di sfruttamento di quel diritto e, inevitabilmente, dai tumultuosi cambiamenti tecnologici, economici e finanche sociali sottostanti.

La natura composita del diritto, nei suoi elementi morali e patrimoniali, l'ambivalenza dei titoli costituzionali invocati, il riferimento naturale alla „Costituzione economica"6, concetto a sua volta oggetto di letture soggette a mutamento, spiegano gli orientamenti non univoci e definitivi della dottrina italiana.

\footnotetext{
${ }^{27}$ Molteplici sono le questioni relative al principio dell'equilibrio di bilancio e al suo rapporto con la tutela dei diritti. La dottrina sul punto è già ampia; ci si limita qui a richiamare P. Bilancia, Riflessioni sulla geografia del potere: poteri, diritti e sistema finanziario, in Rivista AIC, n. 3/2019, 231 ss.; A. Guazzarotti, Poteri, diritti e sistema finanziario tra centro e periferia, in Rivista AIC, $\mathrm{n}$. 3/2019, 291 ss.; G. Rivosecchi, Poteri, diritti e sistema finanziario tra centro e periferia, in Rivista AIC, n. 3/2019, 248 ss.; M. D’Amico, F. Biondi (a cura di), Diritti sociali e crisi economica, Milano, 2017; L. Antonini, Il diritto alla salute e la spesa costituzionalmente necessaria: la giurisprudenza costituzionale accende il faro della Corte, in Federalismi.it, n. 22/2017; M. Luciani, Costituzione, bilancio, diritti e doveri dei cittadini, in AA. VV., Scritti in onore di Antonio d'Atena, III, Milano, 2015.

${ }^{28}$ In questi termini già C. Chiola, La riproduzione „, a stampa“ del pensiero altrui, in Giur. cost., 1976, I, 575.
} 
Autorevole dottrina ${ }^{29}$ ha distinto la garanzia dei diritti morali d'autore dalla componente patrimoniale dello stesso diritto o, meglio, dalle diverse componenti individuate nell' ambito del diritto patrimoniale.

Quanto alla prima voce, il fondamento viene individuato negli artt. 2, 4, 9 e 33 Cost., senza introdurvi elementi di criticità.

Al contrario, quanto al diritto patrimoniale, si è voluto distinguere tra diritto di pubblicazione, fondato sugli art. 21 e 33 Cost.,${ }^{30}$ e il diritto patrimoniale degli autori come diritto esclusivo di sfruttamento dell'opera, quest'ultimo di assai incerta origine quanto a garanzia costituzionale.

Valorizzando la creazione dell'opera quale particolare espressione del lavoro intellettuale, si è invocata a fondamento la norma di cui all' art. 35 Cost., ${ }^{31}$ contenente il principio di tutela del lavoro, in tutte le sue forme e applicazioni“". In questa ricostruzione, dunque, la libera attività di creazione intellettuale trova la sua prima e vera tutela nel riconoscimento del diritto sul risultato creativo. ${ }^{32}$

Va però ricordato che le norme degli artt. 1, 4 e 35 Cost. sono state per lo più lette nella prospettiva della necessaria protezione del lavoratore quale soggetto debole della dinamica economica. Una chiave ricostruttiva forse lontana dalla ratio di tutela dei diritti sui beni immateriali.

Tuttavia, come vedremo, la stessa Corte costituzionale ha avuto modo di richiamare 1'art. 35 quale fondamento, insieme ad altri parametri, della garanzia della libera attività di creazione intellettuale. ${ }^{33}$

Ponendo attenzione anche all'esperienza di altri ordinamenti europei - e, in particolare, alla tradizione tedesca - in dottrina si è ritenuto che l'art. 42 Cost. legittimi e garantisca il sistema di attribuzione di un diritto esclusivo sull'opera dell'ingegno a determinati soggetti, mentre l'art. 35 Cost. possa piuttosto rilevare sul diverso piano dell' appartenenza di questi diritti, legittimando e ,forse addirittura garantendo costituzionalmente" la loro attribuzione all' autore dell'opera. ${ }^{34}$

Secondo la medesima ricostruzione però le norme di legge ordinaria che prevedono le utilizzazioni libere e, in diverso modo, limitano l'esclusiva degli autori dovrebbero essere valutate nella loro legittimità adottando quali parametri non solo l'art. 42 Cost., ma anche le norme di cui agli artt. 9 e 33 Cost., ${ }^{35}$ facendo così ancora

\footnotetext{
${ }^{29}$ L.C. Ubertazzi, voce „Diritto d'autore“, in Digesto delle Discipline Privatistiche-Sezione Commerciale, IV, Torino, 1989, 364 ss., in particolare 370 ss.

${ }^{30}$ L.C. Ubertazzi, voce „Diritto d'autore“ cit. 371. Per il fondamento di questo elemento del diritto sull'art. 21 Cost., si veda anche C. Chiola, La riproduzione cit., 576.

${ }^{31}$ G. Oppo, Creazione ed esclusiva nel diritto industriale, in Riv. Dir. Comm., 1964, I, 187 ss.; e Id., Conversazione sul diritto industriale, in AIDA, 2002, 337 ss.; E. Santoro, Note introduttive sul fondamento costituzionale della protezione del diritto d'autore, in Dir. aut., 1975, 307 ss.; Id., Diritto d'autore e libertà di manifestazione del pensiero (spunti di ricerca), in Dir. aut., 1979, 754 ss.

${ }^{32} \mathrm{G}$. Oppo, Creazione ed esclusiva cit.

${ }^{33}$ Corte cost., sent. n. 108 del 1995, di cui si dirà diffusamente a breve nel testo.

${ }^{34}$ L.C. Ubertazzi, voce „Diritto d'autore“ cit. 371.

${ }^{35}$ L.C. Ubertazzi, voce „Diritto d'autore“ cit. 371.
} 
una volta emergere l'ambivalenza soprattutto del primo dei due articoli ora richiamati, evocato tradizionalmente per fondare il diritto dell'autore.

Come si è anticipato, la stessa Corte costituzionale per lungo tempo non ha affrontato in maniera espressa le complesse questioni sottostanti alla tutela della proprietà intellettuale.

Solo nella sentenza n. 108 del 1995 si trovano osservazioni più diffuse sul fondamento costituzionale del diritto d'autore e sulla composizione dei diversi interessi.

La Consulta, con la pronuncia ora ricordata, ha dichiarato non fondata una questione di costituzionalità sollevata dal Tribunale di Genova in merito alla pretesa del titolare dei diritti patrimoniali d'autore di impedire il noleggio di esemplari legittimamente acquistati, pur in presenza di praticabili soluzioni dirette ad assicurare all'autore di trarre un equo compenso da tale attività.

Il giudice rimettente aveva ipotizzato che il diritto esclusivo dell' autore in ordine al noleggio dell'opera, in primo luogo, potesse irragionevolmente menomare il diritto degli utenti allo sviluppo della persona umana e della cultura, che la Repubblica si impegna a promuovere, si sensi degli artt. 3 e 9 Cost.; comportasse poi la mortificazione della libertà di iniziativa economica privata, sancita dell'art. 41 Cost.; e, infine, limitasse i diritti dell' acquirente, senza peraltro assicurare la funzione sociale della proprietà privata, come stabilito dall' art. 42 Cost.

La Corte - svolta una ricognizione degli interessi considerati nella disciplina normativa posta al suo vaglio e, dunque, operata la ,topografia del conflitto“6, di cui si è detto supra - ha sottolineato che a fronte degli interessi sia del pubblico degli utenti delle opere di cultura che degli operatori economici del settore, rilevano gli interessi dell'autore delle opere. Questi ultimi vengono ritenuti meritevoli di specifica tutela dal legislatore, che sembra affermare un principio di priorità di quegli stessi interessi. ${ }^{36}$

La Consulta riconduce la scelta legislativa, nella sua origine storica al principio personalista, prima ancora che alla promozione della cultura, a cui è chiamata la Repubblica.

Afferma il giudice delle leggi: „La protezione dei diritti patrimoniali e non patrimoniali derivanti da ogni produzione scientifica, letteraria e artistica viene giustificata, per tradizione ormai secolare, dal doveroso riconoscimento del risultato della capacità creativa della personalità umana, cui si collega l'ulteriore effetto dell'incoraggiamento alla produzione di altre opere, nell'interesse generale della cultura".

Si noti che la tutela della persona è invocata dalla Consulta per entrambi i profili dei diritti degli autori: quello morale e quello patrimoniale. Sembra, dunque, emergere una matrice unitaria nella ragione della garanzia costituzionale.

Utilizzando i criteri del bilanciamento tra valori e i criteri della ragionevolezza, la Corte, pur affermando la centralità della protezione della dimensione personale del diritto, valuta il punto di equilibrio individuato dal legislatore per contemperare i diversi interessi.

\footnotetext{
${ }^{36}$ D. Sarti, nota a Corte cost. 6 aprile 1995, n. 108, in AIDA, 1995, 357 ss., criticamente afferma che la pronuncia della Corte ,premette che la legge d'autore non trascura di operare un bilanciamento tra valori ed interessi contrapposti, ma non esplica quali sono questi interessi“; tuttavia, dalla lettura della pronuncia che si è ora riportata nel testo questa individuazione non sembra essere meramente apparente.
} 
Per far ciò la Consulta evidenzia le diverse componenti del diritto d'autore collocandoli nella logica relazionale dei diritti: „La tutela del diritto d'autore - da riferire alle opere dell'ingegno di carattere creativo prodotte mediante le diverse forme in cui si sviluppa l'espressione artistica, e non riducibile ad un mero diritto a compenso - acquista rilievo prevalente rispetto ai diritti degli altri soggetti, pur meritevoli di adeguata tutela in un corretto equilibrio di interessi'“.

La Corte vaglia, dunque, la scelta legislativa, evidenziando come essa operi ,un bilanciamento tra valori ed interessi contrapposti“" nello stesso riconoscimento in capo all'autore della proprietà dell'opera e del suo diritto allo sfruttamento economico della stessa in qualsiasi forma e modo.

Il bilanciamento operato dal legislatore viene giudicato ,non irragionevole“", in quanto realizzato in sintonia con i principi costituzionali ,sia in ordine alla tutela della libertà dell' arte e della scienza (art. 33), sia in materia di tutela della proprietà, da riferire anche all'opera intellettuale (art. 42), sia di tutela del lavoro in tutte le sue forme, tra cui deve farsi rientrare anche la libera attività di creazione intellettuale (art. 35). Tale bilanciamento risulta nel contempo positivamente finalizzato, mediante l'incentivazione della produzione artistica, letteraria e scientifica, a favorire il pieno sviluppo della persona umana (art. 3) ed a promuovere lo sviluppo della cultura (art. 9)“6.

Dette finalità, che indicano la stretta connessione tra tutela degli autori e tutela della cultura, sono ritenute ragionevolmente conciliabili, da un lato, con la libertà dell'iniziativa economica di altri soggetti, quali produttori, rivenditori e noleggiatori, ,in un equilibrio che tenga conto dei rispettivi costi e rischi“' e, dall'altro lato, con i diritti di tutti alla fruizione dell'opera artistica e con l'interesse generale alla diffusione della cultura.

La Consulta ha, quindi, ritenuto che il ,bilanciamento tra valori ed interessi contrapposti'“, compiuto dalla legge nel riconoscere in capo all'autore la proprietà dell'opera ed il suo diritto allo sfruttamento economico della stessa, sia, in sintonia con i principi costituzionali“", di cui agli artt. 33, 42, 35, 3 e 9 Cost.

Sempre sulla scorta della pronuncia della Corte, in dottrina si è tentata una ricostruzione del contrasto fra i diritti esclusivi ed i valori tutelati dall'art. 41 Cost., facendo entrare in gioco però un altro titolo tradizionalmente invocato - l'art. 42 Cost. - , ma secondo una lettura diversa ed evolutiva. ${ }^{37}$

In questa prospettiva i diritti esclusivi potrebbero essere costituzionalmente legittimi se ed in quanto fondati su ulteriori norme costituzionali che giustifichino compressioni alla libera iniziativa economica altrui. Una possibilità rinvenuta, appunto, nell'art. 42 Cost., poiché la norma afferma che la proprietà privata è riconosciuta e garantita dalla legge che ne determina, tra l'altro, ,,i limiti allo scopo di assicurarne la funzione sociale e di renderla accessibile a tutti“6.

Nella ricostruzione però entra in gioco la libera concorrenza, che sarebbe da armonizzare e non da contrapporre al limite costituzionale della ,funzione sociale“6,

\footnotetext{
${ }^{37} \mathrm{~F}$. Benatti, La Street Art musealizzata tra diritto d'autore e diritto di proprietà, in Giurisprudenza commerciale, n. 5/2017, 781 ss., che riprende il pensiero di G. Scaccia, Il bilanciamento degli interessi in materia di proprietà intellettuale, in AIDA, 2005, 198.
} 
che legittima interventi limitativi della proprietà (art. 42 Cost.), nonché a quello di ,utilità sociale“6, che delimita la libertà di iniziativa economica privata, di cui all' art. 41 Cost., a sua volta presupposto necessario per assicurare un impiego commerciale al bene immateriale. ${ }^{38}$

La conciliabilità tra diritto d'autore e libertà di iniziativa economica privata, evocata dalla sentenza della Corte costituzionale, è stata quindi ritenuta possibile grazie ad una lettura in chiave concorrenziale della clausola generale dell' , ,utilità sociale“" che giustifica la compressione della libertà di iniziativa economica privata quando ciò costituisca il costo dell'innovazione culturale, o comunque di una scelta di politica economica che oggi privilegia la dinamicità rispetto alla cristallizzazione delle posizioni acquisite. ${ }^{39}$

La potenziale collisione tra valori costituzionali nella materia che qui ci occupa si arricchisce, dunque, di sempre diverse prospettive. Non solo perché vengono inseriti, o fatti emergere, nuovi valori nel catalogo costituzionale, come la tutela della concorrenza, e in quanto si affermano regole, che finiscono per incidere nelle dinamiche di realizzazione dei diritti, come nel caso dell'introduzione delle norme sull'equilibrio di bilancio a seguito della legge costituzionale n. 1 del 2012, ma anche per la semplice evoluzione concreta di dinamiche in sé risalenti.

È il caso della sempre complessa relazione tra la libertà di manifestazione del pensiero, di cui all'art. 21 Cost., e la tutela dei diritti sui beni immateriali. ${ }^{40}$

È noto che la libertà di espressione ha cessato di rappresentare un mero strumento per impedire la repressione della manifestazione del pensiero nei rapporti verticali tra individuo e Stato ed ha assunto gradualmente il ruolo di ordinatore delle aspettative di accesso alla conoscenza anche nei rapporti tra privati.

Il tema riguarda aree del diritto in grande evoluzione: la disciplina della proprietà intellettuale è divenuta, da regolazione dei rapporti relativi allo sfruttamento delle opere creative, a strumento di protezione (anche) di innovazioni tecniche (come software e banche dati), fino a rappresentare un mezzo idoneo a conformare i comportamenti dell'individuo come singolo e ,nelle formazioni sociali ove si svolge la sua personalità“", come nel caso dei ,,social network ${ }^{66}{ }^{41}$

Si è sostenuto che la proprietà intellettuale e la libertà di espressione siano diritti fondamentali che si trovano in interferenza permanente. ${ }^{42}$ Una interferenza che non viene meno in presenza di norme contenenti eccezioni e limitazioni alla privativa e

\footnotetext{
${ }^{38} \mathrm{G}$. Scaccia, Il bilanciamento cit.

${ }^{39}$ Si veda F. Benatti, La Street Art cit.

${ }^{40}$ Sulla legittimità costituzionale delle norme sul diritto d'autore in riferimento all'art. 21 Cost. si vedano P. Barile, Libertà di manifestazione del pensiero, Milano, 1975, 43, in termini sostanzialmente analoghi, ID, voce Libertà di manifestazione del pensiero, in Enc. Dir., XXIV, Milano, 424 ss.; C. Chiola, La riproduzione cit., 576; E. Santoro, Diritto d'autore e libertà di manifestazione del pensiero cit., 754 ss.

${ }^{41} \mathrm{~A}$. Ottolia, L'interferenza permanente fra proprietà intellettuale e libertà di espressione nel diritto dell'Unione Europea: una proposta di bilanciamento, in AIDA, 2016, 159. La formula utilizzata nel testo richiama volutamente il testo di cui all' art. 2 Cost. In tema ID, L'utilizzo computazionale dell'opera dell'ingegno in internet, in AIDA, 2014, 386 ss. e Privacy e social networks: profili evolutivi della tutela dei dati personali, in AIDA, 2011, 372.
}

${ }^{42} \mathrm{~A}$. Ottolia, L'interferenza cit., 157 ss. 
che - per quanto si è detto supra rispetto alla ricostruzione del sistema dei valori costituzionali - non può essere risolta sulla base di una gerarchia assiologica.

L'interprete, quindi, anche in questo caso sarà sempre chiamato a elaborare un giudizio di bilanciamento, che dovrà però essere condotto sulla base della riduzione al minimo dei sacrifici dei due diritti, sulla scorta di una valutazione di realtà, mentre non potrà essere inteso come realizzazione assoluta di un diritto prevalente.

Nell'eseguire la ricostruzione soccorre il ruolo che il principio personalista svolge anche nella tutela della libertà di manifestazione del pensiero. Tanto che anche in passato il complesso rapporto tra la libertà in parola e il diritto d'autore veniva lucidamente ricomposto alla luce della ,proprietà ${ }^{6 /}$ del pensiero e, dunque, in considerazione del valore costituzionale della persona. ${ }^{43}$

\section{La tutela della proprietà intellettuale nel tortuoso processo evolutivo della libertà di concorrenza}

Quanto si è detto in ordine al fondamento costituzionale della tutela dei diritti sui beni immateriali deve essere collocato nel processo di modifica subito dalle libertà economiche nel nostro sistema costituzionale.

Il fulcro di questo mutamento profondo si rinviene nelle attribuzioni di significato - radicalmente diverse nel tempo - dell'art. 41 Cost. e, segnatamente, per ciò che più qui ci interessa, del limite dell'utilità sociale posto alla iniziativa economica privata dal secondo comma della disposizione ora citata.

La formula, a cui sono stati riconnessi i più diversi e antitetici contenuti - per cui, ad esempio, secondo alcuni essa starebbe ad esortare il raggiungimento dei massimi livelli di occupazione ${ }^{44}$ mentre, secondo altri, finirebbe per essere espressione del principio utilitaristico ${ }^{45}$ - è stata dai più considerata irriducibilmente poliedrica $\mathrm{e}$ indeterminata. ${ }^{46}$ Simbolo della mancanza di una chiara scelta da parte del Costituente, celata dietro il velo della Costituzione economica mista. ${ }^{47}$

\footnotetext{
${ }^{43}$ E. Santoro, Diritto d'autore e libertà di manifestazione del pensiero cit., 754 ss., che sviluppa gli spunti di C. Chiola, La riproduzione cit., 576.

${ }^{44} \mathrm{C}$. Mortati, Il diritto al lavoro secondo la Costituzione della Repubblica, in Problemi di diritto pubblico nell'attuale esperienza costituzionale repubblicana. Raccolta di scritti, III, Milano, 1972, 141 ss.

${ }^{45}$ Secondo la formula di Bentham della maggior quantità di benessere per il maggior numero possibile di uomini. Sulle diverse letture indicate nel testo si veda R. Niro, sub art. 41, in R. Bifulco, A. Celotto, M. Olivetti (a cura di), Commentario della Costituzione, Torino, 2006, 846 - 863.

${ }^{46}$ Per una ricostruzione delle diverse riflessioni dottrinali su tale indeterminatezza si vedano M. Giampieretti, sub art. 41 in S. Bartole, R. Bin (a cura di), Commentario breve alla Costituzione, Padova, 2008, 403 ss.; R. Niro, sub art. 41 cit.; G. Salerno, sub art. 41 in V. Crisafulli, L. Paladin (a cura di), Commentario breve alla Costituzione, Padova, 1990, 287 ss.; G. Mordidelli, voce Iniziativa economica privata, in Enc. Giur., XVIII, Roma, 1989, 1 ss.; F. Galgano, sub art. 41, in G. Branca (a cura di), Commentario della Costituzione, Bologna-Roma, 1982, 11 ss.; A. Baldassarre, voce Iniziativa economica privata, in Enc. Dir., XXI, Milano, 1971, 582 ss.

${ }^{47}$ In questi termini cfr. F. Cintioli, L'art. 41 della Costituzione tra il paradosso della libertà di concorrenza e il , ,diritto della crisi“", in Diritto e società, 2009, 373 ss. Sul concetto di Costituzione
} 
Essa ha comunque operato come un ,principio-valvola“, in grado di consentire l'adattamento dell' ordinamento al mutare dei fatti sociali, ricostruito come un ,concetto valore“" intriso di ,giustizia sociale", che partecipa dei caratteri dei valori costituzionali che connotano l'ordinamento, teso, dunque, a realizzare il progetto di trasformazione della società italiana voluto dal secondo comma dell' art. 3 Cost. ${ }^{48}$

È stato in tal modo individuato un „,nucleo minimo“ del concetto di utilità sociale corrispondente al complesso dei valori che la Costituzione protegge con norme specifiche, ${ }^{49}$ così che sarebbero di utilità sociale quei beni che non solo sono ritenuti tali dal legislatore, ma che godono anche e soprattutto di diretta protezione e garanzia in Costituzione. ${ }^{50}$

Tuttavia, nella giurisprudenza costituzionale si sono viste emergere nel tempo logiche ben diverse, finendosi per ricondurre all' ,utilità sociale“ interessi attinenti alla sfera squisitamente economica.

Si è finito con l'intendere l'utilità sociale come una sorta di ,ordine pubblico economico6, come l'insieme delle ragioni della produttività e dell'efficienza del sistema economico nazionale - in ultima analisi, le ragioni del ,mercato“" - elevate a ragioni giustificatrici del sacrificio delle sfere di libertà del soggetto, ivi compresa la libertà economica.

Per quanto autorevole dottrina abbia dubitato che in Assemblea Costituente il mercato venisse contemplato nell' ambito della tutela costituzionale riservata all'iniziativa economica, ${ }^{51}$ è prevalsa l'opinione incline ad un'interpretazione evolutiva dello stesso art. 41 Cost., capace di comprendere nel perimetro della norma le ragioni ad esso riferibili.

Il rafforzarsi del processo di integrazione comunitaria ha determinato la necessità di leggere le disposizioni costituzionali dedicate alla materia economica in maniera conforme ai principi europei a partire dalla disposizione citata.

Si è così proposto di rintracciare nelle maglie dell' art. 41 Cost il riconoscimento costituzionale della concorrenza o del mercato concorrenziale, superando anche l'ancoraggio alla specifica formula, limitativa, dell'utilità sociale. ${ }^{52}$ Una ricostruzione che troverebbe riscontro già nella legge n. 287 del 1990, recante ,,norme per la tutela della concorrenza e del mercato“, poste ,in attuazione dell'art. 41 Cost. a

economica e sulla sua evoluzione a seguito del processo di integrazione europea e della globalizzazione la bibliografia è, come è noto, sterminata. In questa sede ci si può limitare a rinviare a S. Cassese, La nuova costituzione economica, Bari-Roma, 2019; P. Bilancia, L'effettività della Costituzione economica nel contesto dell'integrazione sovranazionale e della globalizzazione, in Federalismi.it, n. speciale 5/2019.

${ }^{48}$ M. Luciani, La produzione economica privata nel sistema costituzionale, Padova, 1983, 117.

${ }^{49} \mathrm{P}$. Cavaleri, Iniziativa economica privata e Costituzione vivente, Padova, 1978, 37.

${ }^{50} \mathrm{M}$. Luciani, La produzione cit., 138.

${ }^{51} \mathrm{G}$. Amato, Il mercato nella Costituzione, in Quaderni cost., 1992, 7 ss.; N. Irti, L'ordine giuridico del mercato, Bari, 1998, 18 ss.

${ }^{52} \mathrm{La}$ disciplina della concorrenza trova fondamento nel limite dell'utilità sociale di cui all'art. 41, comma 2, Cost. secondo G. Oppo, L'iniziativa economica, in AA.VV., La Costituzione economica a quarant'anni dall'approvazione della Carta fondamentale, Milano, 1990, 70. Per un'analisi di una lettura opposta dei rapporti tra concorrenza e finalità sociali M. Libertini, I fini sociali come limite eccezionale alla tutela della concorrenza: il caso del Decreto Alitalia, in Giur. cost., 2010, IV, 3296 ss. 
tutela e garanzia del diritto di iniziativa economica“6, per quanto in dottrina si sia anche dubitato che il fondamento delle previsioni sulla Autorità antitrust fosse da rintracciare nella disposizione costituzionale citata - o in altre previsioni della nostra Costituzione -, rinvenendosi, piuttosto, direttamente nei trattati europei. ${ }^{53}$

Pur registrandosi, dunque, autorevoli posizioni differenti è opinione diffusa da tempo che l'art. 41 Cost. costituisca il fondamento della libertà di concorrenza, come libertà per certi versi conseguente alla configurazione della libertà di iniziativa economica quale libertà valevole erga omnes.

Una libertà da potersi invocare cioè non solo nei confronti dello Stato e dei pubblici poteri, ma anche nei confronti degli altri privati, così da qualificarla quale ,eguale possibilità “ di tutti ,di attivarsi materialmente e giuridicamente nello stesso settore $^{6}$ e quindi ,di confrontarsi vicendevolmente, sottoponendo al giudizio del mercato la valutazione, e il conseguente successo, delle reciproche iniziative, necessariamente sempre nuove e diverse, in una competizione senza fine ${ }^{66} .54$

Si è diffusamente rilevato in dottrina che solo con l'adesione dell'ordinamento italiano a quello comunitario, ed in particolare con gli sviluppi di quest'ultimo, si sono forniti robusti strumenti di tutela della concorrenza. Di certo, con il citato processo di integrazione si sono definitivamente abbandonate quelle letture - del resto in netto declino a partire dalla fine degli anni settanta del novecento - dei limiti posti all'iniziativa economica privata dai commi secondo e terzo dell'art. 41 Cost. dirette a sostenere il fondamento costituzionale di forme di collettivizzazione o volte ad enfatizzare la necessità dell'intervento diretto dello Stato nell'economia. ${ }^{55}$

È evidente che una tale prospettiva evolutiva passa attraverso il riconoscimento del mutato ruolo della concorrenza nel testo costituzionale, sia a seguito del processo di integrazione europea, sia, testualmente, grazie alla riforma del Titolo V, Parte II, della Costituzione e, segnatamente, all'inserimento della sua tutela - quale bene, fine o valore - tra le materie di competenza esclusiva statale, di cui al secondo comma dell' art. 117 Cost. ${ }^{56}$

Tale percorso interpretativo - che si snoda lungo traiettorie non sempre identiche, muovendo dalla libertà di concorrenza e passando attraverso il mercato concorrenziale comunitario e la concorrenza tout court di cui al nuovo art. 117 - ha coin-

\footnotetext{
${ }^{53}$ B. Caravita, Il fondamento costituzionale della concorrenza, in Federalismi.it, Editoriale, 19 aprile 2017, n. 8/2017.

${ }^{54}$ A. Pace, Gli aiuti di Stato sono forme di „tutela“ della concorrenza?, in Giur. cost., 2004, 262 ss.

${ }^{55} \mathrm{P}$. Bilancia, L'effettività cit., sottolinea come alcune norme della Costituzione economica siano sostanzialmente disapplicate, avendo dovuto cedere il passo alla primazia del diritto europeo. Oltre alle norme relative all'intervento statale nell'economia, di cui agli artt. 41 e 43 Cost., anche quelle dedicate alla tutela del risparmio, contenute nell'art. 47 Cost., sono state profondamente incise. In tema F. Sciuto, La tutela costituzionale del risparmio negli anni della crisi economica. Spunti per un rilancio della dimensione oggettiva e sociale dell'art. 47 Cost., in Federalismi.it, n. speciale 5/2019; P. de Giola Carabellese, Bail-in diritti dei creditori e Costituzione italiana: un recente provvedimento del Consiglio di Stato, in corso di pubblicazione in Giur.comm.

${ }^{56}$ In tema si può qui sinteticamente rinviare ai contributi contenuti in M. Ainis, G. Pitruzzella (a cura di), I fondamenti costituzionali della concorrenza, Bari-Roma, 2019.
} 
ciso con una diversa declinazione della concorrenza nella stessa giurisprudenza costituzionale. ${ }^{57}$

E, infatti, se è possibile rinvenire in alcune decisioni più risalenti della Corte la qualificazione della concorrenza quale libertà che ,integra la libertà di iniziativa economica che spetta nella stessa misura a tutti gli imprenditori“6 ed è ad un tempo ,diretta alla protezione della collettività, in quanto l'esistenza di una pluralità di imprenditori, in concorrenza tra loro, giova a migliorare la qualità dei prodotti ed a contenerne i prezzi“" o ancora quale ,valore basilare della libertà di iniziativa economica $[\ldots]$ funzionale alla protezione degli interessi dei consumatori"6, è nelle decisioni più recenti che sembra sfumare il richiamo alla concorrenza nei termini di libertà ed accentuarsi invece una diversa ,dimensione“6 della medesima nozione proprio sulla suggestione delle indicazioni comunitarie.

Nella emblematica sentenza n. 14 del 2004 la Corte, infatti, non solo afferma che ,dal punto di vista del diritto interno la nozione di concorrenza non può non riflettere quella operante in ambito comunitario, che comprende interventi regolativi, misure antitrust e misure destinate a promuovere un mercato aperto e in libera concorrenza“", ma precisa anche che la tutela della concorrenza ,costituisce una delle leve della politica economica statale e pertanto non può essere intesa soltanto in senso statico, come garanzia di interventi di regolazione e ripristino di un equilibrio perduto, ma anche in quell' accezione dinamica, ben nota al diritto comunitario, che giustifica misure pubbliche volte a ridurre squilibri, a favorire le condizioni di un sufficiente sviluppo del mercato o ad instaurare assetti concorrenziali“". Con ciò adombrando l'idea di una valenza oggettiva del mercato concorrenziale, inerente non più tanto o comunque soltanto alle sfere di libertà dei soggetti (comprensive della libertà di concorrenza, intesa come una sorta di ,altra faccia“ della libertà di iniziativa economica dei soggetti) quanto al sistema economico oggettivamente considerato, in linea con quel principio ,ordinatore“ della Comunità di un',,economia di mercato aperta e in libera concorrenza“, per la realizzazione del quale sembra pertanto possibile il sacrificio o comunque la compressione delle medesime sfere di libertà economica consacrate dall'art. 41 Cost. ${ }^{58}$

La penetrazione della prospettiva europea della tutela della concorrenza nel sistema costituzionale italiano rischia però di determinare letture paradossali, in cui la regolazione del mercato finisce per rendere recessive le sfere di libertà di iniziativa economica.

Proprio per evitare esiti interpretativi di tal genere, in dottrina ${ }^{59}$ si è correttamente ricordato che il rapporto tra l'art. 41 e le norme del Trattato sulla tutela della

\footnotetext{
${ }^{57}$ Come rileva R. Niro, sub art. 41 cit.

${ }^{58} \mathrm{R}$. Niro, sub art. 41 cit. Per l'evoluzione della giurisprudenza costituzionale sul tema cfr. M. Libertini, La tutela della concorrenza nella Costituzione. Una rassegna critica della giurisprudenza costituzionale italiana dell'ultimo decennio, in Mercato Concorrenza Regole, n. 3/2014; Servizio Studi - Corte costituzionale, L. Delli Priscoli (a cura di) La concorrenza, luglio 2015, in www. cortecostituzionale.it.

${ }^{59}$ F. Cintioli, L'art. 41, cit., 373 ss.
} 
concorrenza dovrebbe essere descritto come quello che corre tra un fenomeno soggettivo ed uno oggettivo.

La norma costituzionale fonda una situazione soggettiva di libertà individuale, mentre il diritto comunitario esprime prevalentemente un modello di relazioni economiche e giuridiche. La prima garantisce tutela al diritto di impresa, ma non si impegna nella scelta di sistema che sarebbe stata necessaria per porre liberalizzazione e mercato alla base delle relazioni di tipo economico. La seconda pone, invece, l'apertura del mercato e la libera competizione come premessa per il loro svolgimento.

L'attenzione sull'art. 41 Cost. si è andata spostando dal polo soggettivo della garanzia individuale di una libertà al polo funzionale ed oggettivo del libero mercato concorrenziale come valore in sé, tutelabile in virtù di un suo valore ontologico anziché per la sua strumentalità e soddisfare le pretese dei singoli. ${ }^{60}$

Va, quindi, ribadito che il valore di una libera concorrenza in chiave oggettiva non è valore costituzionale in senso assoluto e che non è riscontrabile la prevalenza della concezione oggettiva su quella soggettiva delle libertà economiche. Pertanto, una legislazione che, in nome della concorrenza, finisca per comprimere in termini assoluti, $\mathrm{o}$, comunque, non ragionevoli e proporzionati, il piano delle libertà individuali non può dirsi conforme a Costituzione.

Anche la tutela della proprietà intellettuale ed i suoi limiti sono stati collocati nel processo evolutivo sopra descritto, che da un'iniziale irrilevanza della concorrenza nelle originarie letture della Carta, o da una sua declinazione in senso addirittura antimercantile, passa attraverso un' interpretazione della concorrenza quale modello economico di mercato, fino ad ampliarne la rilevanza al di fuori del mercato stesso, per assumere, infine, natura di principio di struttura del nostro ordinamento.

Proprio disegnando questa evoluzione, si ritiene, quindi, che il valore della concorrenza ,possa essere considerato, in alcuni ambiti, un criterio di ordinazione del conflitto tra diversi interessi rilevanti“6, che permette, in particolare, ,una rilettura dei limiti generali della proprietà intellettuale che tenga conto anche del valore della concorrenza'6.61

In una tale ricostruzione l'insieme dei principi costituzionali così emergenti dovrebbe autorizzare ,una valorizzazione dello scrutinio antitrust della legislazione sulla proprietà intellettuale ${ }^{66}{ }^{62}$

D'altro canto, la dottrina industrialista ${ }^{63}$ che ha esaminato il rapporto tra diritti di proprietà intellettuale e concorrenza da tempo propugnava un'interpretazione dei primi come coerente ai principi di concorrenzialità, rinvenendo nelle posizioni monopolistiche - tra le quali si deve includere quella che il diritto d'autore riconosce al titolare del diritto e che impedisce iniziative economiche concorrenti - un sacrificio che il legislatore stesso pone alla concorrenza - intesa come possibilità di iniziative

\footnotetext{
${ }^{60}$ F. Cintioli, L'art. 41 , cit.

${ }^{61} \mathrm{G}$. Scaccia, Il bilanciamento cit.

${ }^{62} \mathrm{G}$. Scaccia, Il bilanciamento cit.

${ }^{63}$ D. Sarti, Antitrust e diritto d'autore, in AIDA, 1995, 105 ss.
} 
economiche alternative aventi ad oggetto il medesimo risultato - in cambio del vantaggio di cui la collettività potrà godere, ritenuto a priori socialmente utile.

Tuttavia, è essenziale distinguere le misure limitative della libertà di iniziativa economica ispirate ad una regolazione neutrale e pro-concorrenziale dalle norme che sono, invece, limitative e conformative, ispirate al contemperamento di questa libertà con altri valori costituzionali.

Il fondamento costituzionale dei diritti sui beni immateriali risulta piuttosto il punto di incontro di questo bilanciamento, non olisticamente riconducibile ai principi di concorrenzialità e di mercato.

Del resto, nel mercato - sistematicamente collocato nella trama costituzionale rimane ferma la centralità della persona e, dunque, dei suoi diritti e delle sue libertà.

\section{La radice costituzionale della tutela dei diritti sui beni immateriali}

Le riflessioni svolte sul fondamento costituzionale dei diritti sui beni immateriali hanno fatto chiaramente emergere la difficoltà della stessa individuazione dei titoli di tutela degli interessi in potenziale contrasto, prima ancora che la loro armonica ricomposizione.

Si tratta di profili irti di difficoltà spesso aggirati o sottaciuti. La materia (se così la si può definire, data l'eterogeneità delle sue componenti), del resto, è oggetto di discipline e profili regolatori estremamente complessi; un ambito di normazione molto articolato, prima ancora che a livello interno, secondo una dimensione europea e sovranazionale. In tutta evidenza poi, le norme dedicate alla tutela dei diritti sui beni materiali rientrano tra quelle che maggiormente risentono del tumultuoso sviluppo tecnologico, economico e sociale, che caratterizza i nostri giorni, secondo dimensioni storicamente inedite.

Si osserva comunemente in proposito come la formulazione di qualsiasi disposizione in un quadro di tal genere appaia sempre fuori tempo e tardiva rispetto alla realtà che vorrebbe regolare. Lo stesso contenuto delle norme, dunque, risulta complessivamente inadeguato.

Finanche i principi e i valori costituzionali, non soltanto le disposizioni subordinate, sono toccati da un'evoluzione molto più rapida rispetto al passato, in ragione di fenomeni quali il processo di integrazione europea, la più stretta relazione tra $i$ mercati o l'abbattersi delle gravi crisi finanziarie. ${ }^{64}$

In tale contesto, le tecniche di giudizio della Corte costituzionale, il bilanciamento dei diritti e l'applicazione del principio di ragionevolezza, sembrano un utile strumento anche per una ricostruzione della tutela dei diritti sui beni immateriali, capace di salvaguardare il nucleo essenziale dei valori in gioco.

\footnotetext{
${ }^{64}$ Sulla ricorrenza ciclica delle crisi si veda E.F. Gerding, Law, Bubbles, and Financial Regulation, London and New York, 2016.
} 
Del resto, lo stesso giudice delle leggi italiano negli ultimi anni ha rivendicato per sé un ruolo da protagonista nei meccanismi di protezione dei diritti fondamentali e, prima ancora, ha voluto riaffermare il ruolo centrale della Costituzione. Il nuovo indirizzo giurisprudenziale sui casi di doppia pregiudizialità, costituzionale e comunitaria, coniato con l'obiter contenuto nella nota sentenza n. 269 del 2017 e confermato in recentissime pronunce, ${ }^{65}$ è finalizzato proprio a ridare spazio al giudizio accentrato di legittimità costituzionale, ${ }^{66}$ che aveva rischiato di essere marginalizzato. ${ }^{67}$

La Corte ${ }^{68}$ sollecita i giudici a sollevare questione di legittimità costituzionale ,laddove una legge sia oggetto di dubbi di legittimità tanto in riferimento ai diritti protetti dalla Costituzione italiana, quanto in relazione a quelli garantiti dalla Carta dei diritti fondamentali dell'Unione europea ${ }^{66}{ }^{69}$ Ciò in ragione del , ,contenuto di impronta tipicamente costituzionale“ di quest'ultima, del suo probabile intersecarsi con le previsioni della Costituzione italiana e della conseguente necessità di garantire una pronuncia erga omnes.

La Consulta rivendica probabilmente la centralità del proprio ruolo anche perché l'accertamento di violazioni della Carta di Nizza, proprio in ragione del carattere sostanzialmente costituzionale di quest'ultima, richiede e implica l'utilizzo delle

\footnotetext{
${ }^{65} \mathrm{Si}$ vedano le sentenze nn. 20, 63 e 112 del 2019 e l'ordinanza di rinvio pregiudiziale n. 117 del 2019. In tema G. Repetto, Il significato europeo della più recente giurisprudenza della Corte costituzionale sulla „doppia pregiudizialità “ in materia di diritti fondamentali, in Rivista AIC, $\mathrm{n}$. 3/2019, 1 ss.; S. Leone, Il regime della doppia pregiudizialità alla luce della sentenza $n .20$ del 2019 della Corte costituzionale, in Rivista AIC, n. 3/2019, 642 ss.

${ }^{66} \mathrm{Come}$ si è accennato nel testo, la Corte costituzionale sembra complessivamente impegnata a far ritornare centrale la stessa Costituzione italiana. Chiamata a decidere di potenziali violazioni di norme sovranazionali, tra cui, in primo luogo, la CEDU, il giudice costituzionale italiano ha scelto di privilegiare, nell'ordine dell'esame, proprio i parametri interni e di dichiarare assorbiti i motivi di doglianza incentrati sul rapporto con la fonte sovranazionale. Per una riflessione su questa giurisprudenza S. Leone, Il regime cit., 649 e N. Zanon, Corte costituzionale, evoluzione della „coscienza sociale“, interpretazione della Costituzione e diritti fondamentali: questioni e interrogativi a partire da un caso paradigmatico“, in Rivista AIC, n. 4/2017.

${ }^{67}$ I giudici, infatti, avevano quasi finito per escludere dal sistema della tutela dei diritti fondamentali la Corte costituzionale, attraverso una sostanziale riduzione del giudizio di costituzionalità a quello di compatibilità con il diritto UE. In tema la letteratura è molto ampia, si possono qui richiamare A. Barbera, La Carta dei diritti: per un dialogo fra la Corte italiana e la Corte di giustizia, in Rivista AIC, n. 4/2017; G. Scaccia, Giudici comuni e diritto dell'Unione europea nella sentenza della Corte costituzionale n. 269 del 2017, in Oss. cost., n. 2/2018; D. Tega, La sentenza n. 269 del 2017 e il concorso di rimedi giurisdizionali costituzionali ed europei, in Forumcostituzionali.it, 24 gennaio 2018; A. Guazzarotti, Un ,atto interruttivo dell'usucapione“ delle attribuzioni della Corte costituzionale? In margine alla sentenza n. 269/2017, in Forum di Quad. cost., 18 dicembre 2017. ${ }^{68}$ Nella citata sent. n. 269 del 2017.

${ }^{69}$ Nella dottrina che si è occupata del tema - e che si è precedentemente richiamata - si sono poste questioni complesse relativamente all'ordine delle pregiudiziali e alla natura, obbligatoria o facoltativa, di quella costituzionale; ciò anche in ragione dell'evoluzione giurisprudenziale e, in particolare, del diverso tenore della sentenza n. 269 del 2017 rispetto alla sent. n. 20 del 2019.
} 
tecniche complesse tipiche del giudice delle leggi alle prese con il bilanciamento dei diritti. ${ }^{70}$

Sono modalità argomentative e interpretative capaci di cogliere (ed anzi strutturalmente concepite per cogliere) la realtà nel suo divenire, senza limitarsi all'applicazione di un ragionamento sillogistico, unicamente razionale. Ci si apre così al „giudizio di realtà“, tipico della ragionevolezza.

Questo consente una interpretazione capace di comprendere i fatti nel loro sviluppo, ma pur sempre nell'ambito di un ordinamento applicato secondo procedimenti ermeneutici trasparenti e controllabili.

Porre l'attenzione sui principi e sui valori della nostra Costituzione, che sono a fondamento dei diritti oggetto di analisi, costringe a riflettere sul nucleo essenziale della loro tutela collocata nella trama degli altri diritti e delle libertà sanciti dalla Carta.

Obbliga ad una riflessione sulla nostra specifica identità costituzionale spesso dimenticata nella prospettiva della più ampia dimensione sovranazionale e della regolazione dei mercati.

L'intreccio di interessi al centro del quale si trovano i diritti sui beni immateriali si proietta, dunque, in una dimensione dinamica nella cornice costituzionale, che vede valori e principi propri dei diritti e delle libertà, in una prospettiva di relazione.

Nell'impossibilità di individuare un unico fondamento costituzionale per i diritti di cui si ragiona, ma piuttosto dovendosi riconoscere un insieme di titoli che entrano in potenziale contrasto con altri, un criterio fondante e ordinatore può essere individuato nella centralità della persona umana, scolpita dagli artt. 2 e 3 Cost., e, del resto, da tutto l'ordito costituzionale.

A ben vedere, la tutela della persona, la relativa dimensione relazionale $-\mathrm{e}$, infine, sociale - risultano il perno su cui ruotano le argomentazioni della Corte costituzionale quelle rare volte in cui si è dedicata con organicità alle questioni relative ai diritti sui beni immateriali.

Il disposto di cui agli artt. 2 e 3 Cost. risulta il fondamento ultimo del bilanciamento dei diritti di cui quella giurisprudenza è espressione.

In particolare, va ricordato, che il principio di solidarietà di cui all'art. 2 della Costituzione deve essere interpretato non solo in funzione della previsione dei doveri pubblici posti esplicitamente dalla Carta costituzionale, ma anche come riconoscimento al legislatore ordinario (nell'attuazione di quel principio) della facoltà di affievolire situazioni di vantaggio costituzionalmente garantite ai singoli. ${ }^{71}$

Dunque, il bilanciamento svolto dal legislatore, e sindacato dalla Corte, è, in ultima analisi, un modo efficace di dare attuazione al principio di solidarietà.

\footnotetext{
${ }^{70}$ Coglie questo specifico elemento nella giurisprudenza della Corte costituzionale anche S. Leone, Il regime cit., 652.

${ }^{71}$ E. De Mita, Dalla Costituzione la nuova stagione dei doveri, in Dalla Costituente alla Costituzione. Discorsi per il quarantennale della Repubblica e il quarantennale della Costituzione, Udine, 1988, 61. Per A. Predieri, Un diritto solidarista contro le diseconomie esterne, in „Studi \& Informazioni“", 1993, n. 3, 16 ss., il principio di solidarietà fa nascere obblighi sia verso lo Stato che nelle relazioni intersoggettive. In tema cfr. G. Alpa, La persona. Tra cittadinanza e mercato, Milano, 1992, 53 ss.
} 
Tornare a riflettere sul fondamento più alto della tutela dei diritti sui beni immateriali, dunque, non significa per l'interprete perdersi in questioni concettualmente raffinate e finanche inutili rispetto all'urgenza della ricostruzione di un dettato normativo complesso, di cui spesso è costretto ad invocare la riforma. La prospettiva costituzionale consente piuttosto di ragionare sul nucleo essenziale del riconoscimento di quella stessa tutela, trovando un criterio ordinatore utile in un orizzonte giuridico in continuo divenire.

La lettura sistematica che si è proposta risulta chiaramente propria della prospettiva costituzionale: se in tempi recenti essa è stata dimenticata, non è detto che oggi non possa assumere un rilievo diverso, e si dimostri, invece, uno strumento sufficientemente duttile in un'epoca incomprensibile e ingovernabile con la rigidità.

Open Access Dieses Kapitel wird unter der Creative Commons Namensnennung 4.0 International Lizenz (http://creativecommons.org/licenses/by/4.0/deed.de) veröffentlicht, welche die Nutzung, Vervielfältigung, Bearbeitung, Verbreitung und Wiedergabe in jeglichem Medium und Format erlaubt, sofern Sie den/die ursprünglichen Autor(en) und die Quelle ordnungsgemäß nennen, einen Link zur Creative Commons Lizenz beifügen und angeben, ob Änderungen vorgenommen wurden.

Die in diesem Kapitel enthaltenen Bilder und sonstiges Drittmaterial unterliegen ebenfalls der genannten Creative Commons Lizenz, sofern sich aus der Abbildungslegende nichts anderes ergibt. Sofern das betreffende Material nicht unter der genannten Creative Commons Lizenz steht und die betreffende Handlung nicht nach gesetzlichen Vorschriften erlaubt ist, ist für die oben aufgeführten Weiterverwendungen des Materials die Einwilligung des jeweiligen Rechteinhabers einzuholen.

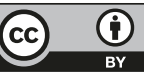

